\title{
Cardiac surgery blues: The midterm impact of postoperative delirium and the association with mood disorders
}

\author{
Juan N. Pulido, MD
}

\footnotetext{
From the Cardiovascular Intensive Care Unit, Cardiothoracic Anesthesiology and Critical Care Medicine, Swedish Heart and Vascular Institute, Swedish Medical Center, US Anesthesia Partners, Seattle, Wash. Disclosures: Author has nothing to disclose with regard to commercial support.

Received for publication Oct 14, 2017; accepted for publication Oct 24, 2017; available ahead of print Dec 9, 2017.

Address for reprints. Juan N. Pulido, MD, Cardiothoracic Anesthesiology and Critical Care Medicine, Swedish Heart and Vascular Institute, Swedish Medical Center, US Anesthesia Partners, WA, 550 17th Ave, No. 680 , Seattle, WA 98122 (E-mail: Juan.Pulido@ Swedish.org).

J Thorac Cardiovasc Surg 2018;155:668-9

$0022-5223 / \$ 36.00$

Copyright (c) 2017 by The American Association for Thoracic Surgery

https://doi.org/10.1016/j.jtcvs.2017.10.067
}

Delirium is an underrecognized but relatively common and costly complication after cardiac surgery. The morbidity and mortality associated with postoperative delirium, ${ }^{1}$ as well as the significant health care costs and resources necessary to care for affected patients, have resulted in an increased interest in understanding this condition and a heightened awareness of its significance. This has led to campaigns with emphasis on early recognition and targeted management of delirium in intensive care units (ICUs) across the United States, regardless of surgical or medical ICU populations. Although most of the of these efforts have traditionally focused on identification of risk factors, prevention, recognition, management, and prognosis, there is growing interest in other aspects of mental health pertinent to quality of life. ${ }^{2}$ There is less known about the long-term mental effects in patients who have delirium after cardiac surgery.

In this issue of the Journal, Nguyen and colleagues ${ }^{3}$ address this knowledge gap. They sought to evaluate the midterm outcomes after postoperative delirium on cognitive and affective functioning in patients after cardiac surgery. The study is a well-executed single-center, prospective cohort study of 197 patients undergoing coronary artery bypass grafting or valve replacement during a period of 12 months. Nguyen and colleagues ${ }^{3}$ focused on understanding the prevalence and significance of delirium, as well as persistent cognitive and affective dysfunction as assessed at 6- to 9-month follow-up during a structured telephone interview.

All patients underwent baseline cognitive functioning and mood assessment with the Montreal Cognitive Assessment and Patient Health Questionnaire-9, respectively. Perioperative data captured major variables, such as pump time, return to the operating room, stroke, cardiac arrest, new dialysis, and acute kidney injury, as well as ICU stay, hospital stay, and mortality. The study carried out a solid protocol that had an organized approach for detection of delirium in the postoperative period, and the midterm outcomes were

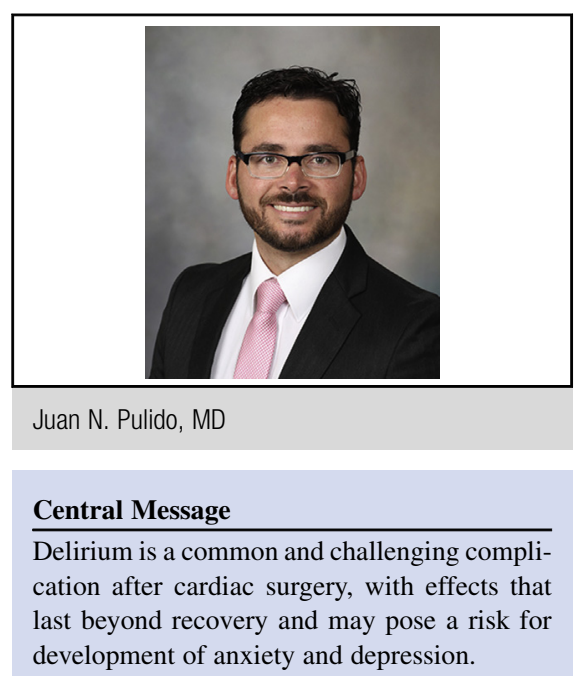

See Article page 660 evaluated with comprehensive recognized and validated assessments (EQ-5D; Patient Health Questionnaire-9; Alcohol Use Disorders Identification Test [AUDIT-C] for affective functioning, substance abuse, and mood evaluation; memory impairment screen and category fluency test assessments for cognition evaluation).

Nguyen and colleagues ${ }^{3}$ found prevalences of delirium of $21.1 \%$ in elective cardiac surgical cases and $30.8 \%$ in the inhouse urgent surgical group. Moreover, age (older), low education (defined as less than high school education), higher euroSCORE II, and previous cardiac procedures were baseline predictors of postoperative delirium. As expected, postoperative complications, such as return to the operating room for bleeding, acute kidney injury, and cerebrovascular accidents, were all associated with increased risk of postoperative delirium. Importantly, but not surprisingly, the presence of delirium at any time during the hospital stay was associated with longer ICU (4 times longer) and hospital ( 5 days longer) stays. During the midterm follow-up evaluation, the presence of delirium was associated with an increased risk of anxiety and depression. Interestingly, no difference in cognitive functioning or alcohol consumption was detected between the delirium and nondelirium groups.

This study offers a unique and powerful message, because all the patients had a baseline cognitive examination, allowing fair comparison and conclusion at midterm 
follow-up and eliminating possible biases related to difference in baseline cognition. Although this study helps in understanding the longer-term effects of delirium after cardiac surgery, however, there are many questions that remain unanswered. What can be done once delirium occurs? Do length, severity, and type (agitated vs hypoactive) of delirium have an effect on outcome? Do early intervention and management make a difference? Does the etiology of delirium matter?

We are just beginning to understand the importance of this complication, including its long-term effects. Further research is definitely needed to evaluate the utility of screening programs for affective disorders and well-being, as well as suitability for psychiatric referral for high-risk patients.

\section{References}

1. Gottesman RF, Grega MA, Bailey MM, Pham LD, Zeger SL, Baumgartner WA, et al. Delirium after coronary artery bypass graft surgery and late mortality. Ann Neurol. 2010;67:338-44.

2. Koster S, Hensens AG, van der Palen J. The long-term cognitive and functional outcomes of postoperative delirium after cardiac surgery. Ann Thorac Surg. 2009;87:1469-74.

3. Nguyen Q, Uminski K, Hiebert BM, Tangri N, Arora RC. Midterm outcomes after postoperative delirium on cognition and mood in patients after cardiac surgery. J Thorac Cardiovasc Surg. 2018;155:660-7.e2. 